\title{
Accurate and precise lattice parameters by selected-area electron diffraction in the transmission electron microscope
}

\section{Enrico Mugnaioli, ${ }^{1, *}$ Giancarlo Capitani,,${ }^{2,} \uparrow$ Fernando Nieto,${ }^{3}$ And Marcello Mellini ${ }^{4}$}

\author{
${ }^{1}$ Institut für Physikalische Chemie, Johannes Gutenberg-Universität Mainz, Welderweg 11, 55099 Mainz, Germany \\ ${ }^{2}$ Dipartimento Geomineralogico, Università di Bari, Campus, Via Orabona 4, 70125 Bari, Italy \\ ${ }^{3}$ Departamento Mineralogía y Petrología and IACT, Universidad de Granata, CSIC, 18002 Granada, Spain \\ ${ }^{4}$ Dipartimento Scienze della Terra, Via Laterina 8, 53100 Siena, Italy
}

\begin{abstract}
Lattice parameters for gold nanocrystals, quartz, and vesuvianite have been determined by electron diffraction in routine transmission electron microscopy (TEM) work, with precision and accuracy near to $0.1 \%$, after correction for elliptical distortion. The distortion, measured in three different microscopes, is constant for each microscope and may be easily eliminated.

Variable camera constants have been avoided by positioning the oriented specimen on the eucentric plane and using parallel illumination. The current flowing in the first intermediate lens was kept fixed, assuring constant conditions of the TEM projecting system, with no further diffraction focus applied.

Application of this method to micas from metamorphic rocks produced deviations between measured and expected values up to $0.8 \%$. Although easy species distinction is still possible, minor crystal chemical differences within the sample may be lost. Likely causes of these deviations are the possible heterogeneous samples, as well as beam damage leading to cation loss with subsequent variation in basal spacings.
\end{abstract}

Keywords: Electron diffraction, lattice parameters, elliptical distortion, mica

\section{INTRODUCTION}

The use of electrons is of the utmost importance when moving from bulk- to nano-characterization, namely with the development of nanosciences. For instance, valuable specimens frequently lack single crystals large enough for X-ray diffraction. Furthermore, from grain to grain they may be significantly heterogeneous, possibly depending on crystallization history.

Two main approaches for structural characterization are possible, based upon direct space and reciprocal space, respectively. The former approach leads to sophisticated high-resolution transmission electron microscopy (HRTEM) techniques, using many tools such as image filtering, image simulation, and image correction based upon experimental contrast transfer function and image correction based upon expensive $\mathrm{C}_{\mathrm{s}}$-corrected lenses. The latter approach emphasizes reciprocal space; namely, it attempts to use electron diffraction as a structural probe, akin to X-ray diffraction. For both the approaches, raw data (lattice images or electron diffraction patterns) may present problems.

A first problem (scale factor) derives from the lack of reliable camera constant values, or image magnification factors. This may lead to the common opinion by which accuracy on the order of only $0.5 \%$ would be possible (Zhang et al. 2002);

\footnotetext{
*E-mail: mugnaiol@uni-mainz.de

$\uparrow$ Present address: Dipartimento di Scienza Geologiche e Geotecnologiche, Università di Milano Bicocca, Piazza della Scienza 4, 20126 Milano, Italy.
}

or, worse, that $L \lambda$ values may change even by a few percent in the same microscope and in the same session. Notwithstanding the existence of previous reports showing case studies with accuracy of $0.1 \%$ (Lodder and Berg 1974) or precision better than $0.05 \%$ (Schamp and Jesser 2005), that pessimistic point of view survives, favored by daily experience with unconstrained measurements, as well as by the known existence of hysteresis effects in the electromagnetic lenses. For the sake of clarity, we recall here that precision is a measure of the standard deviation within a set of measured values, whereas accuracy represents deviation of the measurement from the expected, real value.

A second problem (shape deformation) derives from aberrations present in the TEM column; for instance, projector distortion may transform a circle into an ellipse. To experimentally cope with distortion, Capitani et al. (2006) determined elongation and orientation of the deformation ellipse and proposed a procedure by which distortion was corrected using standard graphic software. This tool is now included in the latest release of the ELD program (Zou et al. 1993). Successful correction may favor quantitative applications of electron diffraction and high-resolution imaging; for instance, measurement of accurate lattice parameters for nanophases or for strained crystals (e.g., Ferraris et al. 2003), or estimation of metric deviations (cell edges and angles) from an assumed symmetry (e.g., Capitani and Mellini 2008).

We now refine the procedure by Capitani et al. (2006) by (1) measuring elliptical distortions in three different laboratories, 
using different detectors and different microscopes; (2) producing accurate calibration of the TEM camera constants; (3) evaluating accuracy and precision of lattice parameters obtained by electron diffraction; (4) checking the reproducibility of the procedure with time; and (5) suggesting simple ways leading to accurate and precise lattice parameters by selected-area electron diffraction (SAED).

We first apply the method to quartz and vesuvianite. Then, we explore the limits of the method dealing with micas in metamorphic rocks. Here, the extreme microstructural diversity often demands the measurement of locally determined lattice parameters. Finally, by analyzing these results, we speculate on the possible drawbacks affecting detailed TEM determinations in the case of beam sensitive materials (Ahn et al. 1986; Noe and Veblen 1999).

\section{EXPERIMENTAL METHODS}

Data were collected in three laboratories (Siena, Bari, and Granada). Siena and Bari have two similar JEOL 2010 microscopes, and Granada a Philips CM-20 microscope (technical data in Table 1). For sake of simplicity, most of the results reported here refer to the camera length of $1200 \mathrm{~mm}$ ( $L \lambda$ close to 30$)$. More data may be found in Capitani et al. (2006).

The amount of elliptical distortion and the camera constant were first evaluated using vacuum-deposited gold nanocrystals on Formvar film. The Au film was approximately $10 \mathrm{~nm}$ thick; the Formvar film was thinner than $20 \mathrm{~nm}$. Gold (cubic, $a=4.0786 \AA$ ) was chosen because of its possible use as an internal standard, when
TABLE 1. Technical data of the three microscopes used for the evaluation of elliptical distortion and camera constants

\begin{tabular}{llll}
\hline & Siena & Bari & Granada \\
\hline Accelerating voltage $(\mathrm{kV})$ & 200 & 200 & 200 \\
Pole piece configuration & UHR & UHR & Twin \\
Resolution $(\AA)$ & 1.9 & 1.9 & 2.7 \\
Holder & Double-tilt & Double-tilt & Double-tilt \\
Recording systems used & Negative films & CCD & CCD \\
& Image plates (IP) & & \\
\hline
\end{tabular}

vacuum-deposited over unknown specimens.

To check the reliability of this calibration, we chose two more high-symmetry reference materials, prepared by ion thinning of standard petrographic thin sections. These were quartz and vesuvianite (trigonal and tetragonal, respectively). The quartz specimen was a (001) section cut from a centimeter-size, transparent crystal; the expected lattice parameter of $a=b=4.91239$ (4) $\AA$ (refined by Will et al. 1988, using synchrotron light) was checked against our own value of 4.9152(2), obtained by conventional X-ray powder diffraction. The vesuvianite specimen was the same (001) section already studied by Capitani et al. (2006); the lattice parameter $a$, refined by X-ray powder diffraction data, is 15.6388 (3). As an internal standard, we vacuum-deposited Au over the quartz and vesuvianite. Figure 1 shows typical SAED patterns for the Au-coated specimens.

Six mica samples were prepared by ion-beam thinning, to apply the method to possibly chemically heterogeneous samples. Three were selected with nearly homogeneous compositions, and consequently nearly constant lattice parameters, and three with heterogeneous compositions (Table 2). Sample P $\beta-94$ is a slate from the South-Portuguese Zone, affected by low-pressure metamorphism reaching the greenschist facies, and containing muscovite with minor illite and moderate $\mathrm{Na}$ content (Abad et al. 2001). Sample 23 is a slate from the West Asturian-Leonese
FIGURE 1. Experimental SAEDs collected on Au-coated samples. (a) Rings of Au nanocrystals vacuum-deposited upon a Formvar film; (b) [001] quartz with $\mathrm{Au}$ rings; (c) [001] vesuvianite with very weak Au rings; (d) [1T0] mica (sample 23) with Au rings.
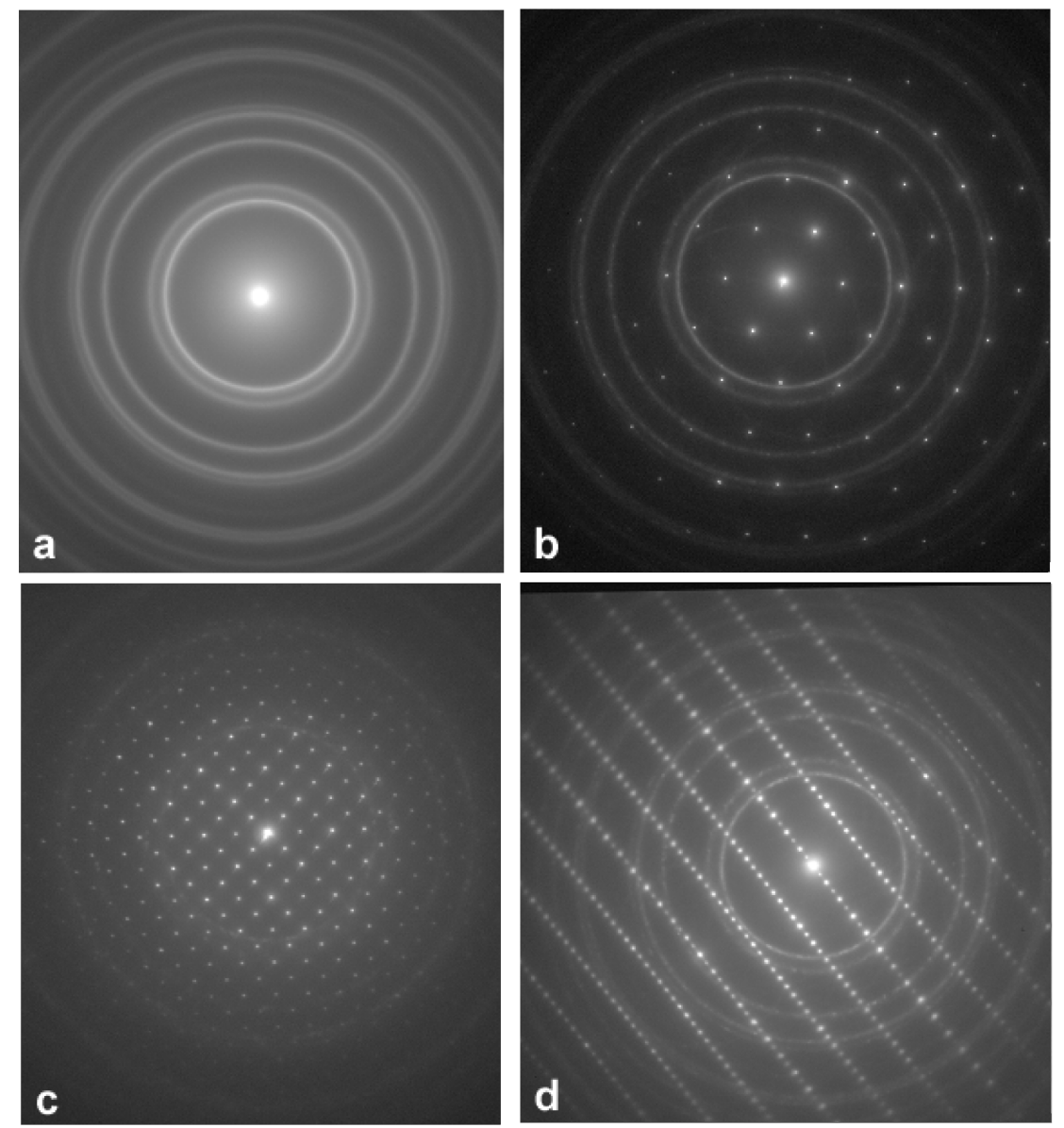
TABLE 2. Lattice parameters (angstroms, by XRD) of the mica samples

\begin{tabular}{lll}
\hline Sample & $b$ & $d_{001}$ \\
\hline Biotite ME-8 & 9.263 & $10.077-10.078$ \\
Muscovite $P \beta-94$ & 8.995 & 9.993 \\
Phengite 23 & 9.022 & 9.971 \\
& & \\
Phengite 16 & 9.033 & 9.965 \\
Muscovite-phengite 4 & $9.009,9.023$ & 9.993 \\
Phengite 34 & 9.027 & 9.962 \\
\hline
\end{tabular}

Zone, affected by intermediate-pressure metamorphism, reaching the epizone (equivalent to greenschist facies). Micas are phengitic, with small but significant illitic substitutions and very low Na content (Abad et al. 2003a). In the two slates, micas have been equilibrated to nearly homogeneous compositions. Biotite ME- 8 was selected as an extreme case of homogeneous composition, due to mediumgrade metamorphism; its higher $b$ and $a$ parameters derive from the trioctahedral nature. Samples 4, 16, and 34 are slates and phyllonites from Talas Ala Tau (Tien Shan, Kyrgyz Republic). They represent extreme cases of heterogeneous mica compositions that result from decompression paths of at least $8 \mathrm{kbar}$. The phyllonitic sample 4 presents two different $b$ values (recognized by XRD). Two populations of intergrown K-dioctahedral micas are recognized in samples 16 and 34, by the different contrast in backscattered electron images (Abad et al. 2003b).

\section{Siena experiments}

SAED patterns were collected at the nominal camera length $L$ of $1200 \mathrm{~mm}$ ( $L \lambda$ close to 30 ), using parallel illumination, with the oriented specimen on the microscope eucentric plane (checked both by specimen tilting and by the value of the objective lens current of $6.94 \mathrm{~A}$ ). The current flowing in the first intermediate lens was kept between 2.62 and $2.64 \mathrm{~A}$, assuring constant conditions of the TEM projecting system (namely, no further diffraction focus was applied). The diffracting area was selected using SAED apertures sampling $0.2,0.5$, or $1 \mu \mathrm{m}$. As we collected our patterns using a low-illumination level (strongly defocused condenser, assuring parallel illumination conditions), the exposure times were larger than in other applications; namely, exposure times were 0.5 to $2 \mathrm{~s}$ in the case of image plates (IPs), up to $22 \mathrm{~s}$ in the case of negative films.

IPs were read using the Fuji FDL5000 scanner, producing files of $3760 \times 3000$ pixels and 16384 gray levels (14 bits), later changed to 8 bits. Negative films were scanned using a photographic transmission scanner CanonScan9900F, producing 8-bit files with 600 dpi resolution; scanned negative films revealed deformation introduced by the photographic scanner, as discussed later.

Ion-thinned specimens produced sharp spots on diffraction patterns. Therefore, measurement of the digitized patterns was undertaken with the ImageProPlus software. In the case of the ring patterns from gold, the origin was determined by a pseudo-diameters method, consisting in the iterative search for the middle points

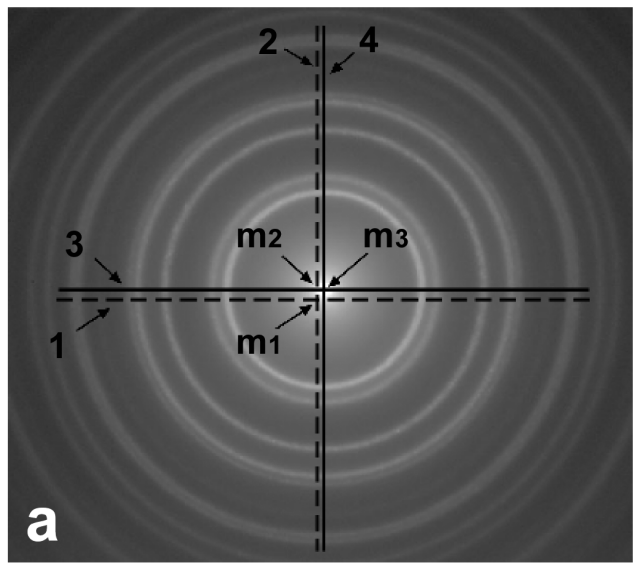

of two orthogonal chords, intersecting in the middle point (Fig. 2a). Finally, ring diameters were measured with the Freehand software (Fig. 2b).

\section{Bari experiments}

Similar experiments were carried out at Bari, with a similar experimental setup. The acquisition device was a Gatan MSC794 CCD, producing digital images of $1024 \times 1024$ pixels and 14-bit gray levels. Experiments were carried out for the entire range of available camera lengths. Cell parameters were measured at different azimuths by the ELD program (Zou et al. 1993). ELD allows an automatic search of the reflection centroids and refines the cell over all the centered reflections.

\section{Granada experiments}

A Philips CM20 microscope was used in Granada, utilizing the same samples and the same analytical protocol. Diffraction patterns were acquired with both CCD camera and photographic films, using camera lengths ranging from 440 to $2350 \mathrm{~mm}$. The CCD was a MegaView III model by SIS with image resolution of $1376 \times 1032$ pixels and 12-bit dynamic range. Here also, we used ELD for the measurements of cell constants at different azimuths.

\section{RESULTS}

\section{Elliptical distortion}

Siena microscope. The distortion was first evaluated from the $\mathrm{Au}$ ring patterns using either the pure gold films or rings from Au-coated [001] quartz. Ring diameters were measured up to the $1.23 \AA$ spacing, for a total of 62 SAED patterns. Data were fitted by least-squares refinement, assuming an elliptical shape. The refined ellipses completely fit the data, confirming that elliptical distortion is the most critical parameter for relatively near-axis diffractions, with respect to barrel, pincushion or spiral distortions. Figure 3, exaggerated, shows two axes of 1.0053 and 0.9947 , respectively, with elongation occurring at $44^{\circ}$ counterclockwise from the long IP side. These values recalculate to the 1:1.0107 ratio, for sake of comparison with the 1:1.0156 ratio measured for the same microscope by Capitani et al. (2006), who found elongation at $60.5^{\circ}$ counterclockwise. However, the fitting method used by Capitani et al. (2006) was slightly different from the present one (instead of fitting the data by a least-squares ellipse, they modeled the deformation factor as a sinusoidal oscillation).

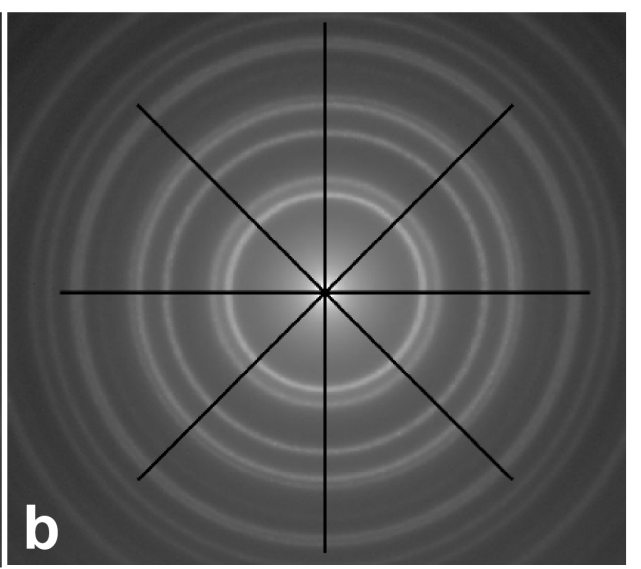

FIGURE 2. Measurement procedure of SAEDs with only Au rings. (a) Procedure for locating the diffraction center. The diffraction center is defined as the middle point of two orthogonal pseudo-diameters. Diameter 1 is drawn parallel to the long IP side and near the diffraction center. Diameter 2 is orthogonal to the diameter 1 and crosses its middle point $\left(\mathrm{m}_{1}\right)$. Then, diameter 1 is translated to the middle point of diameter $2\left(\mathrm{~m}_{2}\right)$ and renamed as 3. Diameter 2 is translated to the middle point of diameter $3\left(\mathrm{~m}_{3}\right)$ and renamed diameter 4 . The procedure goes on until convergence. (b) Ring diameters are therefore measured along four directions at $45^{\circ}$. 


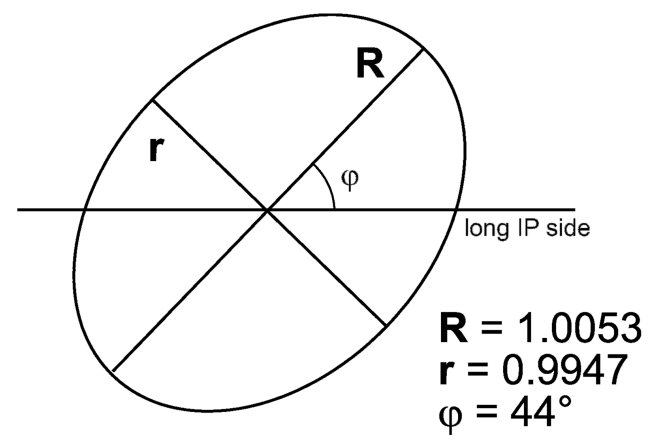

FiguRE 3. Elliptical distortion in the Siena microscope. $R$ and $r$ are the long and short ellipse axes, respectively; $\varphi$ is the angle between the long IP side and the long ellipse axis. The ellipticity of the figure is exaggerated.

Bari microscope. Rather than $\mathrm{Au}$, quartz and vesuvianite were used. Both quartz and vesuvianite indicate a distortion almost constant through the different camera lengths. No significant variations were detected when comparing the distortion ellipse determined from crystals with different orientation within the same TEM mount, i.e., bending effects due to beam pressure or the thin foil's own weight were negligible. Finally, the projector lens distortion was constant over more than one year. Amounts determined from vesuvianite (11/16/2006) and quartz (01/22/2008) for two different camera lengths (Fig. 4) are similar and match the values for the Siena TEM, with major and minor ellipse axes in Bari of 1.008 and 0.992, vs. 1.0053 and 0.9947 now measured in Siena. Conversely, the ellipses are differently oriented, with maxima at $105^{\circ}$ in the Bari TEM.

Granada microscope. In Granada, we were faced with a noteworthy problem. A first data set of diffraction patterns from quartz and vesuvianite were systematically affected by peak oversaturation. The oversaturation may be a problem for automatic measurement of diffraction patterns (as well as for the CCD scintillator), because of the difficulty of locating peak centroids. The problem was more marked in quartz than in vesuvianite. That is readily understood in terms of density of diffraction spots. The [001] orientation of vesuvianite is much denser than that of quartz because of the larger direct space lattice parameters. The diffracted intensity is therefore distributed over a higher number of diffraction spots, making the individual peak oversaturation smaller. We noticed also that automatic measurements are similarly negatively affected by the opposite effect, that is, by low peak to background ratios.

Eventually, choosing the best possible experimental conditions, we were able to measure projector lens distortion on the CCD images from the Philips microscope. It is on average twothree times smaller than in Bari and Siena, with an orientation changing by up to $25^{\circ}$ from camera length to camera length, and on average occurring around $74^{\circ}$. Results for a camera length of $1200 \mathrm{~mm}$ are given in Figure 5.

\section{Correction for elliptical distortion}

The geometrical asymmetry introduced by deformation may be corrected by application of appropriate factors. In the

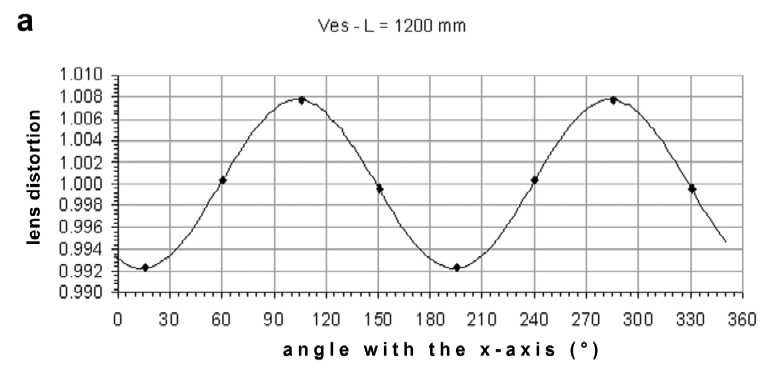

b

$Q t z-L=400 \mathrm{~mm}$

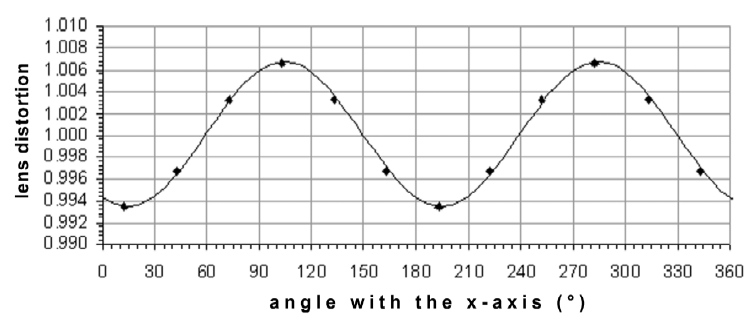

FIGURE 4. Elliptical distortion in the Bari microscope, expressed as sinusoidal oscillation for (a) vesuvianite crystal (dating 11/16/2006) with a nominal camera length of $1200 \mathrm{~mm}$ and (b) quartz crystal (dating $01 / 22 / 2008$ ) with a camera length of $400 \mathrm{~mm}$.

a Ves $-L=1200 \mathrm{~mm}$

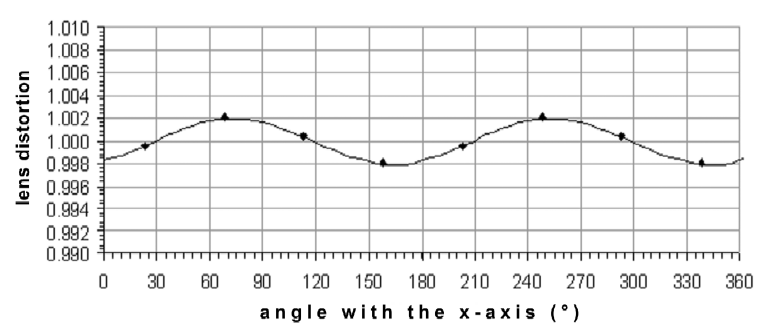

b Qtz $-L=1200 \mathrm{~mm}$

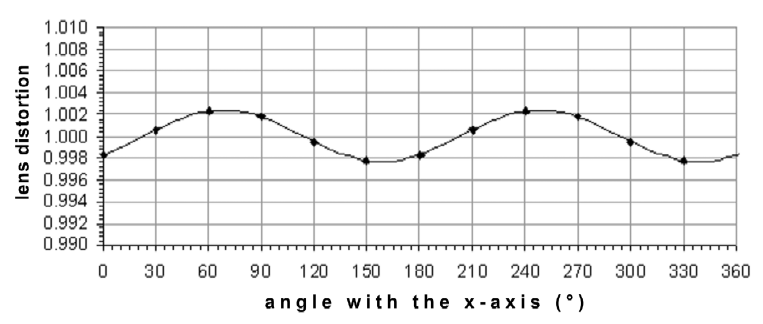

FIGURE 5. Elliptical distortion in the Granada microscope for a camera length of $1200 \mathrm{~mm}$ for vesuvianite (a) and quartz crystals (b).

case of angles, the corrected $\delta_{C}$ value may be obtained by the uncorrected $\delta_{O}$ as:

$$
\delta_{C}=\arctan \left[\frac{R}{r} \tan \left(\delta_{O}-\varphi\right)\right]+\varphi
$$

where $R$ and $r$ are the major and minor ellipse axes, respectively; $\varphi$ is the angle between the major axis and the long IP side. 
Similarly, the observed distance $D_{O}$ may be corrected to $D_{C}$, as:

$$
D_{C}=D_{o} \sqrt{\left[R \sin \left(\delta_{C}-\varphi\right)\right]^{2}+\left[r \cos \left(\delta_{C}-\varphi\right)\right]^{2}}
$$

For $\delta_{O} \approx \delta_{C}$, Equation 2 may be approximated as:

$$
D_{C}=D_{o} \sqrt{\left[R \sin \left(\delta_{o}-\varphi\right)\right]^{2}+\left[r \cos \left(\delta_{o}-\varphi\right)\right]^{2}}
$$

For instance, Table 3 reports the improvement in the measurement precision introduced by the correction factor. Two series of measurements of quartz $d_{100}$ (expected $4.2542 \AA$ ) and one of vesuvianite $d_{100}$ (expected $15.6388 \AA$ ) show internal standard deviations, respectively, of 0.017 and $0.095 \AA$, equivalent to a precision of $0.40-0.61 \%$. After distortion correction, the standard deviations lowered to $0.007 \AA$ for quartz and $0.024 \AA$ for vesuvianite, respectively; this is equivalent to a precision of $0.16-0.15 \%$. For vesuvianite the improvement is more evident because the measured directions are oriented along the main axes of the distortion ellipse.

Of course, the procedure developed here for electron diffraction patterns may be transferred to direct space observations, namely to correct lattice images affected by microscope distortions (e.g., Capitani and Mellini 2008).

\section{Calibration (Siena microscope)}

Electron diffraction is often considered qualitative because of the common belief that $L \lambda$ may change even by a few percent, in the same microscope and in the same session. We suggest that fluctuating values arise from poorly constrained experimental conditions, in agreement with Lodder and Berg (1974).

For instance, care regarding the final specimen position ( $\mathrm{z}$ height) in the microscope is often neglected. This occurs because the defocus introduced by vertical shift of the specimen during alignment is easily compensated by adjusting image focus (namely, operating over the objective lens current), and adjusting the diffraction focus (namely, operating over the intermediate lens current). As a modified lens current is equivalent to a modified magnifying power of the lens, biased data will result from such an unconstrained procedure (Lodder and Berg 1974). For instance, Figure 6 shows the variation of the camera constant, experimentally determined using an internal standard, from the nominal 30 up to 30.5 by adjustment of the intermediate lens current. Therefore, we operated with the oriented specimen on the eucentric plane, verifying also the correct objective and intermediate lens currents.

The calibration was initially performed using the Au film. Due to the possible differences between bulk Au and Au nanocrystals, we checked the lattice parameters, using a double standard, consisting of Au-coated quartz. As the accuracy was on the order of $0.07 \%$, we confidently used the lattice parameter of bulk Au.

By measuring nine different Au SAEDs within the same TEM session, we obtained a camera constant of 30.31(4) (first column of Table 4). On one hand, the camera constant disagrees by $1 \%$ from the nominal value (inaccuracy), thus confirming the need for a careful calibration of the microscope parameters. On the other hand, the values are constant from pattern to pattern (precision), as indicated by precisions slightly larger than $0.1 \%$.
TABLE 3. Observed and corrected measurements on two [001] projec-

\begin{tabular}{|c|c|c|c|c|c|}
\hline \multicolumn{3}{|c|}{ Quartz $d_{100}$} & \multicolumn{3}{|c|}{ Vesuvianite $d_{100}$} \\
\hline Angle & Observed & Corrected & Angle & Observed & Corrected \\
\hline 148 & 4.271 & 4.253 & 135 & 15.759 & 15.680 \\
\hline 148 & 4.281 & 4.262 & 135 & 15.768 & 15.689 \\
\hline 148 & 4.272 & 4.254 & 135 & 15.775 & 15.696 \\
\hline 148 & 4.277 & 4.258 & 135 & 15.779 & 15.700 \\
\hline 88 & 4.250 & 4.252 & 135 & 15.754 & 15.675 \\
\hline 88 & 4.259 & 4.261 & 135 & 15.751 & 15.672 \\
\hline 88 & 4.270 & 4.272 & 135 & 15.783 & 15.704 \\
\hline 88 & 4.265 & 4.268 & 135 & 15.717 & 15.639 \\
\hline 28 & 4.235 & 4.251 & 135 & 15.784 & 15.705 \\
\hline 28 & 4.236 & 4.252 & 135 & 15.727 & 15.648 \\
\hline 28 & 4.237 & 4.253 & 45 & 15.569 & 15.647 \\
\hline 28 & 4.239 & 4.255 & 45 & 15.577 & 15.655 \\
\hline 128 & 4.287 & 4.267 & 45 & 15.599 & 15.677 \\
\hline 128 & 4.283 & 4.263 & 45 & 15.609 & 15.687 \\
\hline 128 & 4.283 & 4.255 & 45 & 15.587 & 15.665 \\
\hline 128 & 4.275 & 4.264 & 45 & 15.571 & 15.649 \\
\hline 68 & 4.250 & 4.265 & 45 & 15.553 & 15.631 \\
\hline 68 & 4.250 & 4.264 & 45 & 15.577 & 15.655 \\
\hline 68 & 4.247 & 4.261 & 45 & 15.573 & 15.651 \\
\hline 68 & 4.250 & 4.265 & 45 & 15.561 & 15.638 \\
\hline 8 & 4.252 & 4.257 & & & \\
\hline 8 & 4.250 & 4.255 & & & \\
\hline 8 & 4.240 & 4.245 & & & \\
\hline 8 & 4.246 & 4.251 & & & \\
\hline Average & 4.258 & 4.258 & Average & 15.669 & 15.668 \\
\hline Std. Dev. & 0.017 & 0.007 & Std. Dev. & 0.095 & 0.024 \\
\hline
\end{tabular}
tions of quartz and one [001] projection of vesuvianite

Notes: First column, angles with the long IP side $\left({ }^{\circ}\right)$; second column, observed measurements; third column, measurements corrected for elliptical distortion. Bottom, average value and standard deviation. After correction, the standard deviation, and therefore the precision of the measurements, are significantly improved. The effect is more evident for vesuvianite, where the measured directions approximately lay along the principal axes of the distortion ellipse. All distances are in angstroms.

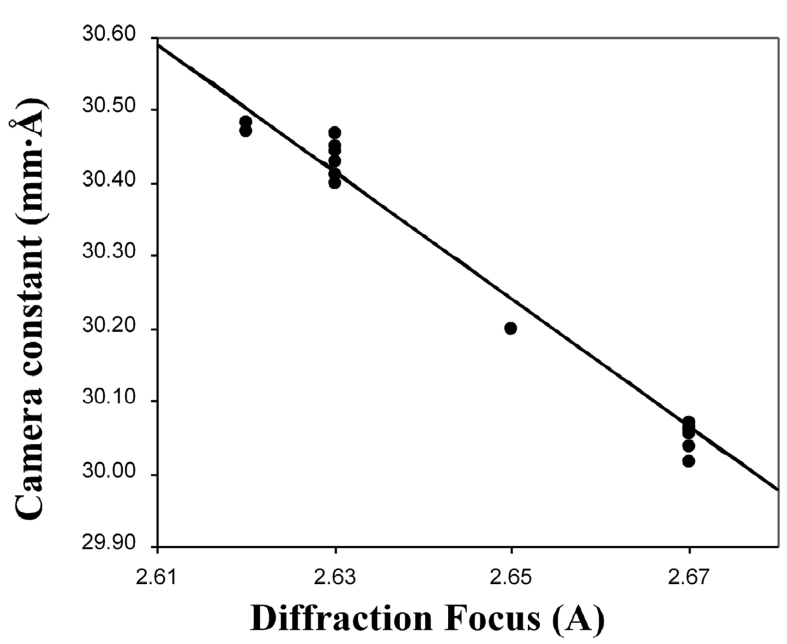

FIGURE 6. Camera constant vs. current in the first intermediate lens (diffraction focus) for Siena microscope.

Similar results were obtained measuring the Au rings in the different samples, at different times (following columns in Table 4). For instance, 6 months later 11 measurements of Au rings from an Au-coated quartz led to a camera constant of 30.44(3).

In agreement with Lodder and Berg (1974) and Schamp and Jesser (2005), small differences were found in the camera constant relative to the distance to the center of the SAED, 
TABLE 4. Camera constants ( $L \lambda$, in angstroms) measured for different SAED on different days and for different samples

\begin{tabular}{|c|c|c|c|c|c|c|c|c|c|}
\hline \multirow{2}{*}{$\begin{array}{l}\text { Date } \\
\lambda \lambda\end{array}$} & $12 / 11 / 06$ & $04 / 12 / 07$ & $04 / 18 / 07$ & $05 / 09 / 07$ & $05 / 09 / 07$ & $05 / 16 / 07$ & $05 / 16 / 07$ & $05 / 30 / 07$ & Overall \\
\hline & $\begin{array}{l}30.345 \\
30.363 \\
30.298 \\
30.341 \\
30.355 \\
30.298 \\
30.285 \\
30.297 \\
30.231\end{array}$ & $\begin{array}{l}30.478 \\
30.501 \\
30.483 \\
30.497 \\
30.517 \\
30.568\end{array}$ & $\begin{array}{l}30.316 \\
30.369 \\
30.336 \\
30.289 \\
30.381 \\
30.365 \\
30.348 \\
30.352 \\
30.288 \\
30.306 \\
30.307 \\
30.348 \\
30.351 \\
30.351 \\
30.356 \\
30.406 \\
30.403 \\
30.371 \\
30.352\end{array}$ & $\begin{array}{l}30.420 \\
30.386 \\
30.430 \\
30.395 \\
30.468 \\
30.378 \\
30.420 \\
30.408 \\
30.477 \\
30.370 \\
30.441 \\
30.377 \\
30.308\end{array}$ & $\begin{array}{l}30.321 \\
30.445 \\
30.410 \\
30.415 \\
30.346 \\
30.334 \\
30.380 \\
30.266 \\
30.309 \\
30.288 \\
30.403 \\
30.349\end{array}$ & $\begin{array}{l}30.285 \\
30.281 \\
30.332 \\
30.267 \\
30.270 \\
30.313 \\
30.314 \\
30.251 \\
30.301 \\
30.302 \\
30.368 \\
30.307 \\
30.360 \\
30.261 \\
30.295 \\
30.252 \\
30.238 \\
30.252\end{array}$ & $\begin{array}{l}30.270 \\
30.225 \\
30.241 \\
30.345 \\
30.305 \\
30.210 \\
30.253 \\
30.235 \\
30.257 \\
30.234\end{array}$ & $\begin{array}{l}30.430 \\
30.446 \\
30.445 \\
30.469 \\
30.484 \\
30.471 \\
30.412 \\
30.450 \\
30.470 \\
30.401 \\
30.412\end{array}$ & \\
\hline Average & 30.312 & 30.508 & 30.347 & 30.406 & 30.355 & 30.292 & 30.258 & 30.445 & $\begin{array}{l}30.365 \\
0.083\end{array}$ \\
\hline
\end{tabular}

Notes: The experimental conditions are similar for different days. Although the standard deviation is lower for measurements made in the same day, with identical experimental conditions, long-term reproducibility is present.

with increasing values for higher distances. As described in the experimental section, our measurement procedure defines the camera constant over all the visible Au rings and refines the cell over all the reflections. Therefore, the camera constant is an average over all the diameter range both for standards and unknowns. However, attention should be paid to such differences if values are obtained using individual reflections; in such a case the reflections used for standards and unknowns need to be at similar distances from the center of the SAED.

Table 4 summarizes calibration reproducibility, reporting data collected at different times, over 6 months, each time using Au rings. During this period, the microscope underwent several alignments; the $\mathrm{LaB}_{6}$ tip was replaced; the emission chamber was opened and the main insulator was exchanged; at least a couple of baking cycles $\left(15 \mathrm{~h}\right.$ at $\left.80-90^{\circ}\right)$ were performed. Notwithstanding, the camera constant did not change significantly. Therefore, we conclude that, even by not performing a specific calibration at the time of measurement, good estimates of lattice parameters (precision of $0.3 \%$ ) may be obtained by using (for instance) the 6-month value of $30.37(8)$.

Applying this calibration for quartz (Table 5), the estimated $a$ lattice parameter would be 4.257(3) Å using either Au as internal standard or an external calibration done on the same day, and 4.246(3) $\AA$ using the 6-month value. Both values closely match the expected $4.2543 \AA$ (Will et al. 1988), with an accuracy of $0.1-0.2 \%$.

For vesuvianite (Table 6), the estimated lattice parameter would be $15.668(16) \AA$ using an external calibration done on the same day, and 15.643(16) $\AA$ using the 6-month value. Both values closely match the value of $15.6388(3) \AA$, refined by X-ray powder diffraction (Capitani et al. 2006). Even by comparing the most mismatched values, the difference is just $0.03 \AA$; so the determination would be accurate by $0.03 / 15.6=0.0019$, close to $0.2 \%$.

\section{Other possible distortions}

During the measurements, inaccuracy may occur at different steps. For instance, our IP trays, used to scan the IPs, host the
TABLE 5. Measurements of quartz $d_{100}(\AA)$

\begin{tabular}{|c|c|c|c|}
\hline & \multicolumn{3}{|c|}{ Quartz $d_{100}$} \\
\hline & Internal standard & Average (1 day) & Average (6 months) \\
\hline & 4.252 & 4.254 & 4.243 \\
\hline & 4.258 & 4.258 & 4.248 \\
\hline & 4.260 & 4.260 & 4.250 \\
\hline & 4.259 & 4.255 & 4.245 \\
\hline & 4.259 & 4.253 & 4.243 \\
\hline & 4.258 & 4.254 & 4.244 \\
\hline & 4.255 & 4.259 & 4.249 \\
\hline & 4.254 & 4.253 & 4.243 \\
\hline & 4.260 & 4.256 & 4.246 \\
\hline & 4.254 & 4.260 & 4.249 \\
\hline & 4.256 & 4.260 & 4.250 \\
\hline Average & 4.257 & 4.257 & 4.246 \\
\hline Std. Dev. & 0.003 & 0.003 & 0.003 \\
\hline \multicolumn{4}{|c|}{$\begin{array}{l}\text { Notes: For different SAEDs, we report the measurements calibrated using Au rings } \\
\text { as internal standard, measurements calibrated with an average camera constant } \\
\text { for that day, measurements calibrated with an average camera constant for } 6 \\
\text { months. The resulting accuracy (difference from observed and expected values) } \\
\text { is } 0.07-0.19 \% \text {. All the measurements are corrected for elliptical distortion. }\end{array}$} \\
\hline
\end{tabular}

IPs within a barely fixed position. The resulting scan may be internally correct, but rotated by an angle up to $5^{\circ}$. This unwanted rotation is evident in the scanned image. If left uncorrected, the rotation will introduce an inaccuracy, of up to $5^{\circ}$, in the distortion ellipse orientation (e.g., Fig. 1d).

Another distortion arises when images are digitalized using a photographic scanner. The amount of distortion may be easily visualized. For instance, in the Siena photographic scanner a perfect circle produces a scanned image other than a perfect circle. To check the amount of the distortion, we picked up a conventional compact disk, acquired its image using our scanner, and applied the same tools used for the analysis of diffraction rings to the scanned images. The fitted ellipse has a major axis of 1.0045 at $8^{\circ}$ from the horizontal.

Care is also necessary if printed material is used, as printers introduce significant distortion.

\section{Illites and biotites}

All the measurements on phyllosilicates (Table 7) are more complex than the ones on $\mathrm{Au}$, quartz, and vesuvianite. Data 
TABLE 6. Measurements of vesuvianite $d_{100}(\AA \AA)$

\begin{tabular}{lcc}
\hline & \multicolumn{2}{c}{ Vesuvianite $d_{100}$} \\
\cline { 2 - 3 } & Average (1 day) & Average (6 months) \\
\hline 15.664 & 15.639 \\
15.672 & 15.647 \\
15.686 & 15.662 \\
15.693 & 15.669 \\
15.670 & 15.645 \\
& 15.661 & 15.636 \\
& 15.667 & 15.643 \\
& 15.647 & 15.622 \\
Average & 15.678 & 15.653 \\
Std. Dev. & 15.643 & 15.619 \\
\hline Notes: For different & 15.668 & 15.643 \\
& 0.0158 & 0.0157 \\
\hline
\end{tabular}

Notes: For different SAEDs, we report the measurements calibrated with an average camera constant for that day and measurements calibrated with an average camera constant for 6 months. The resulting accuracy is $0.03-0.19 \%$. All the measurements are corrected for elliptical distortion.

spreading occurs both deriving the camera constant from the internal standard $(\mathrm{Au})$, or using values merged over different photographs or different days. The range of variation is $0.008-0.045$ $\AA$, much larger than $0.003 \AA$ found for quartz. Variation, higher for the heterogeneous samples (4 and 34), becomes lower for biotite and the nearly homogeneous illitic micas 23 and P $\beta-94$. Nevertheless, the different behaviors of the various samples are not as significant as expected from their chemical homogeneous or heterogeneous character (e.g., sample 16).

Moreover, the average values for all the phyllosilicates deviate substantially from the X-ray diffraction parameters. For dioctahedral micas, all the measured parameters are systematically higher than expected, with expected-observed differences in the range $0.010-0.081 \AA$. Such features contrast with those obtained for quartz and vesuvianite, in the range 0.003 to $0.005 \AA$.

\section{DISCUSSION}

\section{Is the accurate measurement of crystallographic parameters by electron diffraction possible?}

The cases of Au, quartz, and vesuvianite demonstrate the reliable determination of crystallographic parameters by electron diffraction, with precision and accuracy of $0.1 \%$, in agreement with previous conclusions by Lodder and Berg (1974) and Schamp and Jesser (2005). This is an important point, pertinent to future applications to several different minerals. One more result regards hysteresis in the lenses; even if present, hysteresis effects are not so large as to prevent reliable lattice parameter determination.

A trivial application regards the measurement of accurate lattice parameters for minor phases, occurring as tiny crystals or small domains within complex textures. A more interesting application regards the estimate of size-dependent properties, in particular the analysis of the variation of lattice parameters with crystal size, going from bulk material to nanocrystals (e.g., Zhang et al. 2002).

One more stimulating case deals with the distinction of the inner, bulk crystal from its outer region, such as the one occurring close to the crystal surface, or close to internal interfaces and intergrain boundaries. For instance, Ferraris et al. (2003) discussed the occurrence of anomalous lattice parameters for clinoenstatite domains hosted within calcic clinopyroxene, suggesting that the observed anomalous values did not arise from biased determinations. Conversely, these values were interpreted as real intrinsic features of nanometric pyroxenes, indicative for the occurrence of important elastic strain.

In all those examples, the possibility of obtaining data accurate and precise at the $\pm 0.1 \%$ level evidently offers exciting research possibilities.

\section{Application to phyllosilicates in rocks}

The results for the phyllosilicates (Table 7) show larger scattering of data, in a way not univocally related to sample heterogeneity. Furthermore, the data may be far from expected values. So, results for phyllosilicates are apparently worse both in precision and accuracy, with errors larger than for the other materials. In addition, the deviation is significantly higher for $\mathrm{P} \beta-94$ than for 23, notwithstanding that the two samples had been selected as nearly homogeneous. Different explanations may be invoked: (1) the expected values, determined by X-ray diffraction, could be erroneous. This explanation does not hold, as the electron diffraction data are outside of the known range of the mica parameters, and still more for our particular chemical compositions of micas (e.g., Guidotti 1984; Guidotti et al. 1992). (2) Because micas are compositionally heterogeneous (Abad et al. 2006), their lattice parameters are variable within the sample. The parameters obtained by X-ray powder diffraction are an average measurement of the various grains in the powder. This is a known fact and one of the reasons for this study. In fact, the standard deviation among the different grains in the sample is smaller for homogeneous samples than for heterogeneous ones but only two of the three heterogeneous samples conform to that rule (Table 7). (3) Micas are highly anisotropic and sensitive to the electron beam. Electrons in the microscope may easily weaken interlayer cohesion, due to their low bond strength. This would mean that the error should affect the $d_{001}$ parameter, more than the $b$ parameter. Noe and Veblen (1999) studied in detail the development of mottled contrast in

TABLE 7. Expected vs. observed cell measurements for phyllosilicates $(\AA)$

\begin{tabular}{|c|c|c|c|c|c|c|c|c|}
\hline \multirow[t]{2}{*}{ Sample } & \multicolumn{2}{|c|}{ Expected } & \multicolumn{2}{|c|}{ Observed: internal standard } & \multicolumn{2}{|c|}{$\begin{array}{c}\text { Observed: average } \\
\text { camera constant ( } 1 \text { day) }\end{array}$} & \multicolumn{2}{|c|}{$\begin{array}{c}\text { Observed: average } \\
\text { camera constant ( } 6 \text { months) }\end{array}$} \\
\hline & $d_{001}$ & $b$ & $d_{001}$ & $b$ & $d_{001}$ & $b$ & $d_{001}$ & $b$ \\
\hline Biotite & 10.077 & 9.263 & $10.048(16)$ & $9.237(15)$ & $10.053(12)$ & $9.233(15)$ & $10.041(12)$ & $9.222(15)$ \\
\hline Illite $P \beta-94$ & 9.993 & 8.995 & $10.074(24)$ & $9.062(30)$ & $10.066(13)$ & $9.069(14)$ & $10.071(13)$ & $9.074(14)$ \\
\hline Illite 23 & 9.971 & 9.022 & $10.031(20)$ & $9.042(22)$ & $10.043(22)$ & $9.032(15)$ & $10.050(22)$ & $9.038(15)$ \\
\hline Illite 16 & 9.965 & 9.033 & $10.057(17)$ & $9.066(12)$ & $10.074(8)$ & $9.054(13)$ & $10.029(8)$ & $9.014(13)$ \\
\hline Illite 4 & 9.993 & 9.023 & $10.047(45)$ & $9.064(27)$ & $10.054(36)$ & $9.056(23)$ & $10.080(36)$ & $9.079(23)$ \\
\hline Illite 34 & 9.962 & 9.027 & $10.023(31)$ & $9.056(20)$ & $10.027(26)$ & $9.055(19)$ & $10.065(26)$ & $9.088(19)$ \\
\hline
\end{tabular}


TEM images of mica crystals, suggesting the possible existence of beam-induced changes in mica. In particular, they reported reduction in $d_{001}$ by $\sim 3 \%$ with intense exposure. (4) Loss of alkali cations is well known in analytical electron microscopy (e.g., Van der Pluijm et al. 1988), strongly affecting micas. After loss of $50 \%$ of the interlayer cations, the composition of a mica grain would have been drastically changed and consequently its crystallographic parameters might be significantly different from the original ones. And (5) minerals, formed away from Earth surface conditions, have been equilibrated at pressures and temperatures different from laboratory conditions. Usually, the expansion deriving from decompression is more or less balanced by contraction deriving from cooling. For samples analyzed by $\mathrm{X}$-ray diffraction, most of the analyzed volume is far from the crystal surface; therefore, the integrity of the grains is able to cope with the concerted $P$ and $T$ variations (armoring effect). Such integrity is lost during the TEM preparation. Consequently, the analyzed volume is only a few unit cells away from crystal surface. Such local "decompression" might significantly change the lattice parameters (Ahn et al. 1986) due to the high elasticity of phyllosilicates.

Any of these factors, or a combination of them, might be responsible for the lower accuracy in the phyllosilicate measurements. Further research is necessary to explore the exact nature of the problem and to find possible solutions. At the moment, the persistent level of error still precludes the use of electron diffraction in the study of differences in the cell parameters of micas, related with the usual chemical heterogeneity of low-temperature samples. Nevertheless, the accuracy of the measurements is already more than sufficient to distinguish among the different species of micas. Namely, there is no problem in discriminating dioctahedral from trioctahedral micas; or even muscovite $(\mathrm{K})$ from paragonite $(\mathrm{Na})$ and tobelite $\left(\mathrm{NH}_{4}\right)$, with $d_{001}$ of $10,9.6$, and $10.15 \AA$, respectively. Until now their distinction had been possible only when they were present in the same diffraction photograph, based on the expected distances between spots (e.g., as done for muscovite and paragonite by Giorgetti et al. 1997, or muscovite and tobelite by Nieto 2002).

The techniques described in this paper have the potential to solve many problems connected with closely intergrown textures (e.g., microtwins, strained crystals, anomalous lattice parameters).

\section{ACKNOWLEDGMENTS}

M.M. is grateful to the Secretaría de Estado de Universidades y Investigación del Ministerio de Educacion y Ciencia de España for the invitation to spend a sabbatical term at Universidad de Granada (Grant SAB2005-0191), where this study originated. F.N. is grateful to the financial support by Research Project no. CGL2007-66744 (Ministerio de Educacion y Ciencia de España).

\section{REFERENCES CITED}

Abad, I., Mata, M.P., Nieto, F., and Velilla, N. (2001) The phyllosilicates in diagenetic-metamorphic rocks of the South Portuguese Zone, southwestern Portugal. The Canadian Mineralogist, 39, 1571-1589.

Abad, I., Nieto, F., and Gutiérrez-Alonso, G. (2003a) Textural and chemical changes in slate-forming phyllosilicates across the external-internal zones transition in the low-grade metamorphic belt of the NW Iberian Variscan Chain. Schweizerische Mineralogische und Petrographische Mitteilungen, 83, 63-80.

Abad, I., Gutiérrez-Alonso, G., Nieto, F., Gertner, I., Becker, A., and Cabero, A. (2003b) The structure and the phyllosilicates (chemistry, crystallinity and texture) of Talas Ala-Tau (Tien Shan, Kyrgyz Republic); comparison with more recent subduction complexes. Tectonophysics, 365, 103-127.

Abad, I., Nieto, F., Gutiérrez-Alonso, G., Do Campo, M., Lopez-Munguira, A., and Velilla, N. (2006) Illitic substitution in micas of very low-grade metamorphic clastic rocks. European Journal of Mineralogy, 18, 59-69.

Ahn, J.H., Peacor, D.R., and Essene, E.J. (1986) Cation-diffusion-induced characteristic beam damage in transmission electron microscope images of micas. Ultramicroscopy, 19, 375-382.

Capitani, G.C. and Mellini, M. (2008) Rationale for the existence of 4- and 8reversals in antigorite. American Mineralogist 93, 796-799.

Capitani, G.C., Oleynikov, P., Hovmöller, S., and Mellini, M. (2006)A practical method to detect and correct lens distortion in the TEM. Ultramicroscopy, 106, 66-74.

Ferraris, C., Folco, L., and Mellini, M. (2003) Sigmoidal exsolution by internal shear stress in pyroxenes from chondritic meteorites. Physics and Chemistry of Minerals, 30, 503-510.

Giorgetti, G., Memmi, I., and Nieto, F. (1997) Microstructures of intergrown phyllosilicate grains from Verrucano metasediments (northern Apennines, Italy) Contributions to Mineralogy and Petrology, 128, 127-138.

Guidotti, C.V. (1984) Micas in metamorphic rocks. In S.W. Bailey, Ed., Micas, 13, p. 357-467. Reviews in Mineralogy, Mineralogical Society of America, Chantilly, Virginia.

Guidotti, C.V., Mazzoli, C., Sassi, F.P., and Blencoe, J.G. (1992) Compositional controls on the cell dimensions of $2 M_{1}$ muscovite and paragonite. European Journal of Mineralogy, 4, 283-297.

Lodder, J.C. and Berg, K.G. (1974) A method for accurately determining lattice parameters using electron diffraction in a commercial electron microscope. Journal of Microscopy, 100, 93-98.

Nieto, F. (2002) Characterization of coexisting $\mathrm{NH}_{4}$ - and $\mathrm{K}$-micas in very low-grade metapelites. American Mineralogist, 87, 205-216.

Noe, D.C. and Veblen, D.R. (1999) Mottled contrast in TEM images of mica crystals. American Mineralogist, 84, 1932-1938.

Schamp, C.T. and Jesser, W.A. (2005) On the measurement of lattice parameters in a collection of nanoparticles by transmission electron diffraction. Ultramicroscopy, 103, 165-172.

Van Der Pluijm, B.A., Lee, J.H., and Peacor, D.R. (1988) Analytical electron microscopy and the problem of potassium diffusion. Clays and Clay Minerals, 36, 498-504.

Will, G., Bellotto, M., Parrish, W., and Hart, M. (1988) Crystal structures of quartz and magnesium germanate by profile analysis of synchrotron-radiation highresolution powder data. Journal of Applied Crystallography, 21, 182-191.

Zhang, F., Chan, S.W., Spanier, J.E., Apak, E., Jin, Q., Robinson, R.D., and Herman, I.P. (2002) Cerium oxide nanoparticles: size-selective formation and structure analysis. Applied Physics Letters, 80, 127-129.

Zou, X., Sukharev, Y., and Hovmöller, S. (1993) ELD — a computer program system for extracting intensities from electron diffraction patterns. Ultramicroscopy, $49,147-158$

MANUSCRIPT RECEIVED APRIL 4, 2008

MANUSCRIPT ACCEPTED JANUARY 9, 2009

MANUSCRIPT HANDLED BY LAURENCE GARVIE 